\title{
EDITORIAL
}

\section{Antimicrobial Drug Resistance: Need for An National Policy}

\section{Introduction}

We can not think a world without antibiotic. Since the discovery, antibiotics have proved remarkable effective control of bacterial infections. Antibiotic resistance is one of the world's most serious public health problem. Antibiotic resistance is a consequence of antibiotic use. The introduction of penicillin in the early 1940's dramatically improved the prognosis of infections. However as early as 1942 penicillin resistant Staphylococci were recognized first in hospitals and subsequently in community ${ }^{1}$. By the late 1960 s $80 \%$ of Staphylococcus were resistant to penicillin ${ }^{2}$. Initial problem of bacterial resistance to antibiotics was solved by discovery of new antibiotics. But it appear that new antibiotic can't keep pace with the development of resistance.

There were 16 new antibiotics created by drug companies between 1983 to 1987. Seven new ones from 1998 to 2002 and only 3 new antibiotics from 2003-2008 3 .

Around 350 million US \$ are required from research to marketing of an antibiotic. Rapid development of drug resistance make the drug ineffective. So there is dramatic reduction in Antibiotic research and pharmaceuticals have shifted their research money to newer, safe and lucrative areas (like Viagra) ${ }^{4}$. So far resistance has developed against all antibiotics.

Antibiotics are often prescribed inappropriately and inadequately and have thus become one of the highly abused drugs. The increasing antibiotic resistance is largely due to wide spread and irrational use of antibiotics in hospital and community. Unless antibiotic resistance problems are detected as they emerged and if proper actions are not taken to contain them the world may face with pan resistant bacterial strain with no known antibiotic to combat them.

\section{Background of Antibiotic resistance development}

Antibiotic resistance is a consequence of antibiotic use. Total consumption of antibiotic is the critical factor in the development of resistance. Abuse of antibiotic, easy availability of antibiotic over the counter, inadequate dose and time are mainly held responsible for development of resistance. Over use of antibiotic by the unregistered practitioner also play important role. Except one none of our tertiary level hospitals have antibiotic guide line.
Hospitals are a critical component of antibiotic resistance development. Absence of antibiotic guide line particularly in ICU has worsen the resistance situation ${ }^{5}$. Antibiotic resistance has not only increased mortality but also has increased in health care cost of hospitalized patient with infections. In many cases bias information gathered from the medical representative, particularly in newer antibiotic has unfortunately lead to irrational prescription of Antibiotic $^{6}$.

One study revealed $35 \%$ of antibiotics prescribed on out patient department in USA are unnecessary. In Thailand it is $75 \%{ }^{6}$. No reproducible data is available for us, but assumed that it would be similar to Thailand. In USA 14,000 people die each year from drug resistant infections ${ }^{7}$.

\section{Need for antibiotic prescription guidance}

With the background of increase in antibiotic resistance, which has increased mortality, morbidity and increase in health care cost, there is an urgent need for antibiotic prescription guide line.

Spread of resistance is associated with antibiotic therapy, has stressed the need for rational use of antibiotics.

Absence of large scale surveillance data in Bangladesh has put in difficulties for development of Antibiotic prescription guide line. To determine the resistance pattern there is an absolute and urgent need for nation wide survey for antibiotic susceptibility testing. There is also need to review the antibiotic prescription practices of the clinicians.

We must also address the problem with indiscriminate dispensing of the antibiotics of the pharmacy counters without any licensed prescriptions.

We are looking at containment of resistance not eradication which is impossible.

Following are the area where due importance must be given while developing antibiotic guideline and national policies for the country.

- Restriction in use of antibiotic. Each secondary and tertiary level hospital should have their own Antibiotic prescription guide line based on statistics generated in that hospital and locality. Use of restricted antibiotic guide lines will be a step towards the upgradation in 
the antibiotic polices for of multi disciplinary and tertiary level health care hospital. An antibiotic policy restricts the occurrence of antibacterial resistance among the hospital strains and control spread of such infections to susceptible and critically ill patients in hospital. Antibiotic policy also help in limiting use of powerful antibiotic as initial treatment and made available of such antibiotic for resistant organism and saves life and money. Making provision for written justification while prescribing high cost antibiotic.

- Putting restriction on higher antibiotic to be dispensed only on prescription by specialist/ registered physician.

- Making gradual restriction on over the counter sale of antibiotic without prescription of a registered practitioner.

- Ensure the availability of culture and sensitivity facility upto district level hospital.

- Putting more emphasis on antimicrobial resistance in under and post graduate medical curriculum.

- A nation wide survey should be conducted to develop master antibiogram.

- Multidisciplinary national task force to coordinate policies and strategies to contained antibiotic resistance from related discipline and public representative should be formed for preparing National Antibiotic Prescription policy and recommendations to combat Antimicrobial drug resistance.

- Designing national campaign program to prevent Antiboitc resistance in health care system.

- Development of national antimicrobial resistance monitoring system.

- Setting a national reference microbiology laboratory either separate or department of Microbiology of any national institute/ university for testing antibiotic sensitivity.

- Implementation of infection control programs in hospitals.

- Development of awareness regarding drug resistance among healthcare worker. Only practice of hand washing can reduce infection upto 50\% cases.
- Antibiotic recycling : Antibiotics which has markedly reduced efficacy may be banned and reintroduce after 2/3 years, they may regain the efficacy.

- Control contact of Medical representative with doctor.

- Arrangement of refreshers course on antimicrobial resistance for General physician. May be made mandatory for renewal of BMDC registration.

Overall goals of any antibiotic resistance control program should be to improve antibiotic utilization, reduce antibiotic resistance, improve patient outcomes and reduce antibiotic expenditure.

All infections are potential threat to life. Antibiotic are life saving weapon. Weapon of such importance should be used with all care and precaution. If we abuse them we lose then.

[BSMMU J 2009; 2(1): 1-2.]

\section{Dr. Ahmed Abu Saleh}

Associate Professor

Department of Microbiology and Immunology

Bangabandhu Sheikh Mujib Medical University, Dhaka

E-mail: asaleh@gmail.com

\section{References:}

1. American Society for Microbiology. Report of the ASM task force on antibiotic resistance. Antimicrob. Agents Chemother. 1995 (suppl) 1-23.

2. Joshi MC, Tariq K, Ejaj A. Restricted anti biotic policy; Rational approach towards antibiotic resistance. Policy-Express Healthcare Management. Indian Express, Business Publications Division, Issue dtd. $1^{\text {st }}$ to $15^{\text {th }}$ June, 2005.

3. Coast J, Smith R, Miller MR. Superbugs: should antimicrobial resistance be included as a cost in economic evaluations ? Health economics. 1996; 5: 217-226.

4. Coast J, Smith RD, Millar MR. An economic perspective on policy for antimicrobial resistance. Social Science and Medicine. 1998; 46: 29-38.

5. Antimicrobial resistance on Health and Economic outcomes. Clinical Infect Dis. 2003; 36: 1433-1437.

6. Lacy MK, Klutman NE, Horvat RT, Zapantis A. Antibiograms : New NCCLS guidelines, development and clinical application. Hop Pharm 2005; 43: 2629-34. 